\title{
Amino groups modified SBA-15 for dispersive-solid phase extraction in the analysis of micropollutants by QuEchERS approach
}

\author{
Michele Castiglioni ${ }^{a}$, Barbara Onida ${ }^{\mathrm{b}, *}$, Luca Rivoira ${ }^{\mathrm{a}}$, Massimo Del Bubbac \\ Silvia Ronchetti ${ }^{\mathrm{b}}$, Maria Concetta Bruzzonitia,* \\ a Department of Chemistry, University of Turin, Via P. Giuria 7, 10125 Turin, Italy \\ ${ }^{\mathrm{b}}$ Department of Applied Science and Technology, DISAT, Polytechnic of Turin, Corso Duca degli Abruzzi 24, 10129 Turin, Italy \\ 'Department of Chemistry "Ugo Schiff”, University of Florence, Via della Lastruccia 3, 50019 Sesto Fiorentino, Florence, Italy
}

\section{A R T I C L E I N F O}

\section{Article history:}

Received 2 February 2021

Revised 19 March 2021

Accepted 21 March 2021

Available online 26 March 2021

\section{Keywords:}

QuEChERS food analysis

D-SPE

Sugar removal

SBA-15 mesoporous silica

Functionalization

\begin{abstract}
A B S T R A C T
In the analysis of contaminants in food products, sample preparation is performed by proper adsorbents, whose choice is crucial to eliminate matrix interference. In this work we modified SBA15 adsorbents by functionalization with (3-aminopropyl)-triethoxysilane (SBA-15-APTES) and N-[3(trimethoxysilyl)propyl]aniline (SBA-15-AN) aiming to use them for the first time in the clean-up step of a QuEChERS (quick, easy, cheap, effective, rugged and safe) extraction of micropollutants from strawberry, a sugar rich fruit. After physico-chemical characterization by nitrogen adsorption, infrared spectroscopy and thermogravimetric analysis, the adsorption capabilities of SBA-15 sorbents and possible interaction mechanisms were studied at different $\mathrm{pH}(2.1-8.5)$ for glucose, sucrose and fructose at concentrations characteristic of those found in strawberries. The performance of the two SBA-15 sorbents was compared with that of commercial PSA (primary secondary amine), usually proposed in QuEChERS protocols. Both SBA-15 materials exhibit up to 30\% higher adsorption than PSA, suggesting their possible QuEChERS application. Synthesized SBA-15 adsorbents were hence used as innovative dispersive sorbents in the QuEChERS extractions of 13 PAHs and 14 PCBs from strawberry. For PCBs, SBA-15-AN provides better matrix removal than PSA and comparable extraction recoveries around 90\%. For PAHs, the use of SBA-15-AN has the advantage of lower relative standard deviation (7\%) than PSA (19\%).
\end{abstract}

(C) 2021 Elsevier B.V. All rights reserved.

\section{Introduction}

Fruits and their transformation products (e.g. beverages and jams) may be altered by organic micropollutants due to many contamination sources, such as soil and irrigation water [1,2], as well as packaging materials [3] and pesticide application [4].

Current regulation regarding the presence of organic micropollutants in fruits and vegetables includes polycyclic aromatic hydrocarbons (PAHs), polychlorinated dibenzo(p)dioxins (PC$\mathrm{CDs}$ ), polychlorinated dibenzo(p)furans (PCDFs), and polychlorinated biphenyls (PCBs) with dioxin-like properties, referred to as dioxine-like PCBs (DL-PCBs) [5].

Most of the above-mentioned compounds in fruits and derived products are analysed by using the QuEChERS approach, followed by gas chromatographic-mass spectrometric analysis (GC-MS) $[2,6]$.

\footnotetext{
* Corresponding Author

E-mail addresses: barbara.onida@polito.it (B. Onida), mariaconcetta.bruzzoniti@unito.it (M.C. Bruzzoniti).
}

QuEChERS extraction is based on the partition of target analytes between water and acetonitrile.

Sugars present as natural components or as additives are wellknown interferents in the QuEChERS approach since they are coextracted in acetonitrile and must be removed prior to the analysis of the extracted samples by chromatographic methods.

This task is usually accomplished using solid-phase extraction (SPE) cleanup, even through on-line approaches [7], or by a dispersive solid phase extraction ( $d$-SPE) clean-up step, with a primary secondary amine (PSA) sorbent $[8,9]$. Several manufacturers make commercially available proprietary PSA sorbents which are based on silica chemically modified with ethylenediamine$\mathrm{N}$-propyl group, as bulk packing, or within ready-to-use kits for QuEChERS analysis. PSA is usually claimed to be a sorbent more retentive than aminopropyl phases due to presence of the secondary amine [10], even if dedicated studies on the adsorption mechanisms are not yet available.

Ordered mesoporous silicas and organosilicas have been largely investigated as adsorbents [11-14] due to their high specific surface area and uniform porosity, together with the possibility of 
tailoring their surface chemical properties through synthesis conditions and post-synthesis modification. In particular, in the context of analytical chemistry, ordered mesoporous organosilicas have been tested as adsorbents for food samples cleanup [15].

Mesoporous silica functionalized with amino groups was previously investigated for adsorption and removal of anionic pollutants in wastewater $[16,17]$.

The aim of this work is to synthesize and study the performance of organically modified ordered mesoporous silicas to be innovatively included as $d$-SPE sorbents in a QuEChERS protocol for the removal of co-extracted sugars for the analysis of contamination of strawberries by 13 PAHs and 14 PCBs, including dioxine-like congeners. PCBs and PAHs are often found in fruit $[18,19]$ and are therefore a major concern for health protection.

Ordered mesoporous silica of SBA-15 family was functionalized with the primary amine (3-aminopropyl)-triethoxysilane (APTES) and, in another case, with the secondary amine $\mathrm{N}$-[3(Trimethoxysilyl)propyl]aniline, in order to mimic the commercial PSA. After the determination of the main physico-chemical characteristics, the adsorption of glucose, fructose and sucrose (sugars naturally contained in fruit) was studied. The performances of the two organically modified SBA-15 silica as QuEChERS d-SPE sorbents were investigated determining the signal to noise ratio and the extraction recoveries for target compounds, in comparison with those obtained by commercial PSA.

To the best of our knowledge no reports are currently available on the use of ordered mesoporous organosilicas in the QuEChERS technique.

\section{Materials and Methods}

\subsection{Reagents}

All reagents used were of analytical grade. Ordered mesoporous silica of the SBA-15 type was purchased from ACS Material Advanced Chemical Supplier (Pasadena, CA, USA). (3-aminopropyl) triethoxysilane (APTES, 99\%), N-[3-(Trimethoxysilyl)propyl]aniline, toluene (99.8\%), acetonitrile (>99.9\%) were purchased from Sigma Aldrich (Steinheim, DE). Primary Secondary Amine (PSA, Fig. 1A) was purchased from Agilent Technologies (Santa Clara, CA, USA). $\mathrm{D}(+)$ glucose was purchased from Merck (Darmstadt, DE), D(-) fructose and sucrose were purchased from J.T. Baker (Phillipsburg, NJ, USA).

The PAHs studied were the $13 \mathrm{PAH}$ compounds listed by EPA 525.1 procedure and were purchased from Sigma Aldrich-Merck (Darmstadt, Germany): acenaphtylene (AcPY), fluorene (Flu), phenathrene (Phe), anthracene (Ant), pyrene (Pyr), benzo[a] anthracene $(\mathrm{BaA})$, chrysene $(\mathrm{Chr})$, benzo[b]fluoranthene $(\mathrm{BbFl})$, benzo[k]fluoranthene (BkFl), benzo[a]pyrene (BaP), indeno[1,2,3cd]pyrene (Ind), dibenzo[a,h]anthracene (DBA), benzo[ghi]perylene (BP). PCBs were purchased from LGC Standards (Milan, Italy). They were non-dioxine like PCBs: 3,3'-dichlorobiphenyl (PCB 11), 4,4'-dichlorobiphenyl (PCB 15), 2,4,4'-trichlorobiphenyl (PCB 28), 2,2',5,5'-tetrachlorobiphenyl (PCB 52), 2,2',4,5,5'pentachlorobiphenyl (PCB 101), 2,2',3,4,4',5-hexachlorobiphenyl
(PCB 138), 2,2',4,4',5,5'-hexachlorobiphenyl (PCB 153), 3,3',4,4',5,5'hexachlorobiphenyl (PCB 169), 2,2',3,4,4',5,5'-heptachlorobiphenyl (PCB 180), 2,3,3',4,4',5,5'-heptachlorobiphenyl (PCB 189); and dioxine like PCBs: 3,4,4',5-tetrachlorobiphenyl (PCB 81), 2,3',4,4',5pentachlorobiphenyl (PCB 118), 2',3,4,4',5-pentachlorobiphenyl (PCB 123), 2,3',4,4',5,5'-hexachlorobiphenyl (PCB 167).

Hydrochloric acid (35\% w/w, d = $1.187 \mathrm{~g} / \mathrm{mL}$ ) and $\mathrm{NaOH}(>98 \%)$ were from Carlo Erba (Milano, IT). $\mathrm{NaCl}, \mathrm{MgSO}_{4} \cdot 7 \mathrm{H}_{2} \mathrm{O}$ and $\mathrm{H}_{2} \mathrm{SO}_{4}$ $(95-97 \%, d=1.84 \mathrm{~g} / \mathrm{mL}$ ) were from Sigma-Aldrich. High-purity water $\left(18.2 \mathrm{M} \Omega \cdot \mathrm{cm}\right.$ resistivity at $\left.25^{\circ} \mathrm{C}\right)$, produced by an Elix-Milli $\mathrm{Q}$ Academic system from Millipore (Vimodrone, MI, Italy), was used for standard and eluent preparation.

\subsection{Preparation of sorbents}

The synthesis procedures of the two sorbents were based on previous work concerning on the functionalization of SBA-15 silica $[16,17]$. In a flask, $1 \mathrm{~g}$ of SBA-15, previously washed with about $50 \mathrm{~mL}$ of deionized water, was stirred with $200 \mathrm{~mL}$ of toluene for $30 \mathrm{~min}$, at room temperature. Afterwards, $2 \mathrm{~mL}$ of functionalizing reagent ((3-aminopropyl)-triethoxysilane-APTES or $\mathrm{N}$-[3-(trimethoxysilyl)propyl]aniline, Fig. $1 \mathrm{~B}$ and 1C) were added dropwise. The flask was then connected to a water-refrigerated system and the solution heated to $110^{\circ} \mathrm{C}$. The solution was kept under stirring for 24 hours. Afterwards, the powder was filtered and dried.

Hereafter the sample functionalized with APTES is denoted SBA-15-APTES and the sample functionalized with $\mathrm{N}$-[3(trimethoxysilyl)propyl]aniline is denoted SBA-15-AN.

\subsection{Physico-chemical characterization}

Nitrogen adsorption isotherms were measured using a Quantachrome AUTOSORB-1 (Boynton Beach, FL, USA) instrument. Prior to nitrogen adsorption, samples were outgassed at $393 \mathrm{~K}$ for $6 \mathrm{~h}$. The BET specific surface areas (SSA) were calculated in the relative pressure range from 0.04 to 0.1 and the pore size distribution were determined through the NLDFT (Non Localized Density Functional Theory) method, using the equilibrium model for cylindrical pores.

For FTIR measurements, powders were pressed in selfsupporting wafers and spectra were recorded at room temperature with a Bruker Tensor 27 (Bruker, Billerica, MA, USA) spectrometer operating at $2 \mathrm{~cm}^{-1}$ resolution, after outgassing the sample at room temperature (residual pressure of $0.1 \mathrm{~Pa}$ ).

Thermogravimetric analisys (TG) were carried out between 298 $\mathrm{K}$ and $1073 \mathrm{~K}$ in air (flow rate $100 \mathrm{~mL} / \mathrm{min}$ with a heating rate of $10 \mathrm{~K} / \mathrm{min}$ ) using a SETARAM 92 (Caluire, France) instrument.

Density functional theory (DFT) simulations were calculated by means of Gaussian 09W and Gaussian View (Gaussian Inc, Wallington, US).

\subsection{Chromatographic analysis}

The evaluation of sugar content was performed by ionchromatography using an ICS-3000 gradient pump, Thermo Fisher<smiles>CCO[Si](CCCN)(OCC)OCC</smiles><smiles>CO[Si](C)(CCCNc1ccccc1)OC</smiles>

Fig. 1. Schematic representation of PSA (A) and structure of the precursors APTES (B) and N-[3-(trimethoxysilyl)propyl]aniline (C). 
Scientific, Waltham, MA, USA, coupled to pulsed amperometric detection. An AD40 Electrochemical Detector, Thermo Fisher Scientific, equipped with $\mathrm{Ag} / \mathrm{AgCl}$ reference electrode and a gold working electrode was used. The detection potential was set at $0.1 \mathrm{~V}$ and maintained for $400 \mathrm{~ms}$ : the first $200 \mathrm{~ms}$ represents the delay time and the second $200 \mathrm{~ms}$ represents the determination time. The potential was then instantaneously set at $-2 \mathrm{~V}$ and maintained for $10 \mathrm{~ms}$ and raised at $0.6 \mathrm{~V}$ and maintained for $10 \mathrm{~ms}$ to restore the gold oxide necessary to maintain an active working electrode surface. The potential was finally set at $-0.1 \mathrm{~V}$ and maintained for $60 \mathrm{~ms}$, to reduce the small amount of gold oxide previously formed. The waveform requires a total of $500 \mathrm{~ms}$.

The sample $(10 \mu \mathrm{L})$ was injected through a six-ways Rheodyne injection valve. The column used was a CarboPacPA10, $250 \times 4 \mathrm{~mm}$ (100 $\mu \mathrm{eq} /$ column), Thermo Fisher Scientific which has a microporous substrate with particle size of $10 \mu \mathrm{m}, 55 \%$ cross-linking functionalized with a difunctional quaternary ion latex (5\% crosslinking).

After optimization, the eluent concentration was kept at $55 \mathrm{mM}$ $\mathrm{KOH}$ (data available upon request). At these conditions, limits of detection (LODs) for glucose, fructose and sucrose, calculated as $s_{m}=s_{m}+3 s_{b}$, with $s_{m}=$ average signal of blank, $s_{b}=$ standard deviation of blank on ten measurements, were respectively 69, 56 and $11 \mu \mathrm{g} / \mathrm{L}$. Pump, detector settings, and data collection were managed by the Chromeleon v.6.80 software (Thermo Fisher Scientific).

For PAHs and PCBs analysis, a gas chromatographic-mass spectrometric (GC-MS) method was used, according to previous studies by our research group [2,20], employing an Agilent (Santa Clara, CA,USA) 6980 series gas chromatograph coupled with an Agilent 5973 Network mass spectrometer detector.

The GC column was a (5\%-Phenyl)-methylpolysiloxane column (HP $5 \mathrm{~ms}, 30 \mathrm{~m} \times 0.25 \mathrm{~mm} \times 25 \mu \mathrm{m}$, Agilent), with He as gas carrier $(1 \mathrm{~mL} / \mathrm{min})$. MS detection was performed in Single Ion Monitoring (SIM) mode, selecting for each analyte its proper $\mathrm{m} / \mathrm{z}$ ratio $(\mathrm{m} / \mathrm{z}$ ratio available upon request). $2 \mu \mathrm{L}$ of each sample were injected using the Pulsed Splitless mode (pressure at 40 psi for $2.5 \mathrm{~min}$ ). The oven ramp was: $90^{\circ} \mathrm{C}$, hold for $2 \mathrm{~min}$; ramp to $176^{\circ} \mathrm{C}, 12^{\circ} \mathrm{C} / \mathrm{min}$ rate; ramp to $196^{\circ} \mathrm{C}, 5^{\circ} \mathrm{C} / \mathrm{min}$ rate, hold for 3 mins; ramp to $224^{\circ} \mathrm{C}$, $12^{\circ} \mathrm{C} / \mathrm{min}$ rate; ramp to $244^{\circ} \mathrm{C}, 5^{\circ} \mathrm{C} / \mathrm{min}$ rate, hold for $3 \mathrm{~min}$; ramp to $270^{\circ} \mathrm{C}, 7^{\circ} \mathrm{C} / \mathrm{min}$ rate, hold for $3 \mathrm{~min}$; ramp to $300^{\circ} \mathrm{C}, 5^{\circ} \mathrm{C} / \mathrm{min}$, hold for 10 min to completely clean and restore the GC column. The total run time for the complete separation of PAHs and PCBs is $52 \mathrm{~min}$.

LODs, calculated as previously described for ion chromatography, were in the range from $0.06 \mu \mathrm{g} / \mathrm{L}$ (BaA) to $2.10 \mu \mathrm{g} / \mathrm{L}$ (DBA), while from $0.49 \mu \mathrm{g} / \mathrm{L}$ (PCB153) to $5.40 \mu \mathrm{g} / \mathrm{L}$ (PCB169). Detailed analytical features of the GC-MS method are presented in Table S1 (for PAHs) and S2 (for PCBs) of the Supplementary Information section.

GC-MS data were handled with OpenChrome software (Lablicate, Germany).

\subsection{Adsorption tests}

Tests to evaluate the effect of $\mathrm{pH}$ were performed on $0.25 \mathrm{~g}$ of sorbent. The sorbent was put in contact with $4 \mathrm{~g}$ solution containing $350 \mathrm{mg} / \mathrm{g}$ glucose, $350 \mathrm{mg} / \mathrm{g}$ fructose and $170 \mathrm{mg} / \mathrm{g}$ sucrose. These concentrations were chosen in order to match those contained in strawberry according to Kasperbauer [21]. Solutions were stirred at $1100 \mathrm{xg}$ for $10 \mathrm{~min}$. Experiments were performed at $\mathrm{pH}$ 2.1, 5.0 and 8.5 .

\subsection{Strawberry fruits}

Commercial strawberry samples grown in Italy were used. Fragaria $x$ ananassa, Camarosa cultivar was chosen as model plant, since it accounts for about $60 \%$ of the strawberry world's production and it adapts greatly to wide climate and growth conditions.

\subsection{Extraction of PAHs and PCBs from strawberries by QuEChERS}

Among organic micropollutants, PCBs and PAHs, present in the environment as a result of natural and anthropogenic processes, represent both point source and diffuse emissions [22].

PAHs and PCBs were extracted using a QuEChERS approach [8,23]. Briefly, $5 \mathrm{~g}$ of homogenized strawberries were put in a vial containing $10 \mathrm{~mL}$ acetonitrile: $\mathrm{H}_{2} \mathrm{O} \mathrm{pH} 2.1$ (70:30), $8.2 \mathrm{~g} \mathrm{MgSO}_{4}$ and $1 \mathrm{~g} \mathrm{NaCl}$. The tube was vigorously shaken and centrifuged at $1100 \mathrm{xg}$ for $10 \mathrm{~min}$. After extraction, a $4 \mathrm{~mL}$ aliquot of the supernatant was then transferred in a new vial containing $0.25 \mathrm{~g}$ of sorbent (PSA or SBA-15-APTES or SBA-15-AN) and $1.0 \mathrm{~g} \mathrm{MgSO}_{4}$ for the clean-up step. The tube was shaken and centrifuged (7870 $\mathrm{xg}$, $10 \mathrm{~min}$ ) and the supernatant was directly analysed by GC-MS. For each sorbent, the recovery was calculated spiking the fruit samples prior- (i) and post- (ii) extraction as follows:

- Pre-extraction spike: a cumulative batch of $50 \mathrm{~g}$ of homogenised strawberries was spiked with $6666 \mu \mathrm{L}$ of a solution containing PAHs and PCBs at $300 \mu \mathrm{g} / \mathrm{L}$ each, to achieve a final concentration of $0.04 \mathrm{mg} / \mathrm{kg}$. According to the extraction procedure above detailed, a theoretical final concentration of $20 \mu \mathrm{g} / \mathrm{L}$ is expected for each PAH and PCBs. The procedure was repeated in triplicate.

- Post-extraction spike: after the extraction and clean-up procedure, $187 \mu \mathrm{L}$ of the extract is spiked with $13 \mu \mathrm{L}$ of the solution containing PAHs and PCBs at $300 \mu \mathrm{g} / \mathrm{L}$, so to obtain a final concentration of $20 \mu \mathrm{g} / \mathrm{L}$ for each PAH and PCBs. The procedure was repeated in triplicate.

For each analyte, extraction recovery percentage (ER\%) was calculated from the peak areas obtained after the pre-extraction spike $\left(A_{\text {pre-extraction }}\right)$ and post-extraction $\left(A_{\text {post-extraction }}\right)$ according to the equation:

$\mathrm{ER} \%=100 * \mathrm{~A}_{\text {pre-extraction }} / \mathrm{A}_{\text {post-extraction }}$

For each analyte and experimental conditions, signal-to-noise ratio $(\mathrm{S} / \mathrm{N})$ was derived from the software OpenChrome and was calculated from the height of the analyte chromatographic peak $\left(\mathrm{H}_{\text {peak }}\right)$ and that of the noise $\left(\mathrm{h}_{\text {noise }}\right)$, according to the 2015 United States Pharmacopeia definition [24]:

$\mathrm{S} / \mathrm{N}=2 \mathrm{H}_{\text {peak }} / \mathrm{h}_{\text {noise }}$

\section{Results and discussion}

\subsection{Physico-chemical characterization}

Table 1 reports textural features of SBA-15 as such and of functionalized SBA-15. For both SBA-15-APTES and SBA-15-AN, lower values of SSA, pore volume and pore diameter are observed with respect to the pristine SBA-15. These results reveal that grafting of organic moieties on the internal surface of silica mesopores occurred during functionalization.

Table 1

Textural features of SBA-15, SBA-15-APTES, SBA-15-AN and PSA

\begin{tabular}{lllll}
\hline & SBA-15 & SBA-15-APTES & SBA-15-AN & PSA \\
\hline SSA $\left(\mathrm{m}^{2} / \mathrm{g}\right)$ & 490 & 323 & 298 & 384 \\
Pore volume $\left(\mathrm{cm}^{3} / \mathrm{g}\right)$ & 0.92 & 0.53 & 0.54 & 0.70 \\
Pore diameter $(\mathrm{nm})$ & 8.1 & 7.0 & 6.8 & 7.0 \\
\hline
\end{tabular}




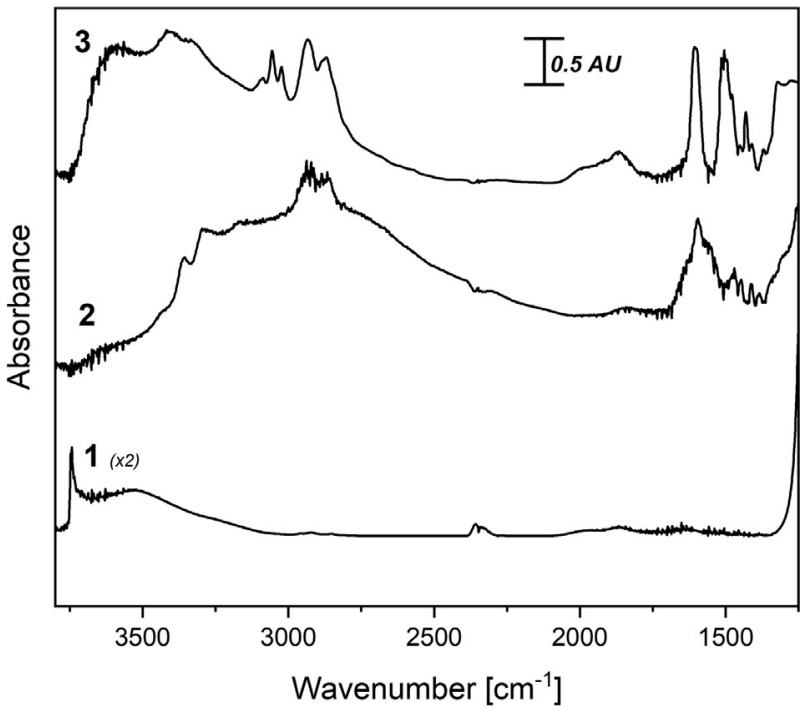

Fig. 2. FT-IR Spectra of SBA-15 (curve 1), SBA-15-APTES (curve 2) and SBA-15-AN (curve 3 ). The spectrum of SBA-15 was amplified by a factor 2 for sake of clarity.

$\mathrm{N}_{2}$ adsorption measurement was carried out also on PSA since no data for the commercial product were available. It is worth noting that the same features of PSA are in between those of SBA-15 and organically modified SBA-15, so that, as a whole, we can consider PSA, SBA-15-APTES and SBA-15-AN comparable systems for the proposed QuEChERS application.

Fig. 2 shows FT-IR spectra of SBA-15, SBA-15-APTES and SBA15-AN after outgassing at room temperature.

The spectrum of SBA-15 (curve 1) reveals the typical features of an amorphous silica, i.e. the narrow band at $3743 \mathrm{~cm}^{-1}$, due to the stretching mode of isolated silanols, and the broad band centred at $3530 \mathrm{~cm}^{-1}$, due to $\mathrm{H}$-bonded silanols.

In the spectrum of SBA-15-APTES (curve 2), bands due to the organic moieties are clearly observed, that are: (i) two bands at $3355 \mathrm{~cm}^{-1}$ and $3295 \mathrm{~cm}^{-1}$ attributed to the stretching modes of $-\mathrm{NH}_{2}$ groups; (ii) two bands at $2930 \mathrm{~cm}^{-1}$ and $2875 \mathrm{~cm}^{-1}$ due to the stretching modes of aliphatic $-\mathrm{CH}_{2}$ - groups; (iii) a band at about $1595 \mathrm{~cm}^{-1}$ due to the bending mode of $-\mathrm{NH}_{2}$ groups. As usually observed for amorphous silica modified with APTES, a broad and ill-defined absorption is observed at about $3000 \mathrm{~cm}^{-1}$, on which the previous mentioned bands are superimposed. This absorption is due to the residual silanols engaged in $\mathrm{H}$-bonding with surface $-\mathrm{NH}_{2}$ groups [25]. Indeed, in the spectrum, the stretching mode of isolated silanols is not present.

In the case of SBA-15-AN (curve 3), as for SBA-15-APTES, the bands due to the organic groups are visible in the spectrum, i.e. (i) the band at $3330 \mathrm{~cm}^{-1}$ due to the stretching mode of -NHspecies; (ii) bands above and below $3000 \mathrm{~cm}^{-1}$ due to, respectively, aromatic $-\mathrm{CH}$ and aliphatic $-\mathrm{CH}_{2}$ - stretching modes; (iii) bands below $1700 \mathrm{~cm}^{-1}$ due to ring modes of the aromatic moieties. Moreover, a broad absorption is observed at about 3600 $\mathrm{cm}^{-1}$ which is tentatively ascribed to the residual silanols engaged in H-bonding with aromatic rings [26].

In summary, the set of IR data reveal that amine species in modified SBA-15 are located and exposed on the silica surface, in agreement with textural features (Table 1) discussed above.

From TG curves (not reported), the amount of amino groups was estimated and it is $4.41 \mathrm{mmol} / \mathrm{g}$ for SBA-15-APTES and 2.23 $\mathrm{mmol} / \mathrm{g}$ for SBA-15-AN.

According to data found for commercially available PSA in the market, capacity for PSA ranges from 0.65 to $1.22 \mathrm{mmol} / \mathrm{g}[27,28]$. The above-mentioned capacity data suggest, per se, a competitive performance of functionalized SBA-15 in respect to PSA, and supports the study herein proposed.

\subsection{Adsorption measurements}

The following experiments were performed in order to optimize the $\mathrm{pH}$ conditions to be used in QuEChERS application. Furthermore, the results may be useful in formulating possible interactions acting between adsorbents and sugars during retention.

Results are represented in Fig. 3a, 3b, 3c. According to the acidic dissociation constants of the sugars considered $\left(\mathrm{pK}_{\mathrm{a}}=12.2\right.$ for glucose, 12.0 for fructose and 12.6 for sucrose), the concentration of dissociated sugars may be considered negligible at $\mathrm{pH}$ values studied (2.1, 5.0 and 8.5).

Organically modified mesoporous silicas show better removal performance than commercial PSA for all the $\mathrm{pH}$ values. It is worth noting that textural properties such as SSA and pore volume of SBA-15-APTES and SBA-15-AN are comparable to those of PSA (see Table 1), therefore the better adsorption performance of organically modified mesoporous silica has to be ascribed to the functional groups acting as adsorption sites, the amount of which is larger in SBA-15-APTES and SBA-15-AN than in PSA. Nevertheless, a role of different surface chemical properties of the two silica supports, cannot be ruled out.

Firstly, the retention properties of aminopropyl-modified silica and PSA towards sugars has been ascribed mainly to H-bonding [29]. The role of H-bonding of protonated ethylenediamine based materials, structurally similar to PSA, in the retention of uncharged compounds is supported by many authors [30,31], who also speculated on possible leading retention mechanisms via $\mathrm{H}$-bonding as a function of $\mathrm{pH}$ and on the abundancy of $\mathrm{NH}_{3}{ }^{+} / \mathrm{NH}_{2}$ and $\mathrm{NH}_{3}{ }^{+} / \mathrm{NH}$ groups [31].

As shown in Fig. 3, for all the adsorbents, retention capabilities decrease at increasing $\mathrm{pH}$. This may be ascribed to a change of the relative population of protonated and deprotonated amine groups and to their involvement in $\mathrm{H}$-bonding. At increasing $\mathrm{pH}$, the population of protonated amines decreases. Since protonated amines are stronger Brønsted acids than amines, they may be considered also stronger $\mathrm{H}$ donor in $\mathrm{H}$-bonding.

Data in Fig. 3a-3b-3c show that SBA-15-AN has higher retention abilities than SBA-15-APTES at all the pH values tested (SBA-15-AN $>$ SBA-15-APTES > PSA). Indeed, if we consider that aniline $\left(\mathrm{pK}_{\mathrm{b}}\right.$ 8.9 ) is a weaker base than propylamine $\left(\mathrm{pK}_{\mathrm{b}} 3.5\right)$, the decrease of protonated amine population upon increasing $\mathrm{pH}$ is expected to be relatively larger in SBA-15-AN than SBA-15-APTES. Thus, it may be proposed that $\mathrm{H}$-bonding between protonated amines and sugar molecules, where the former act as proton donor and the latter as proton acceptor, does not play the only role in the adsorption.

Therefore, the better performance of SBA-15-AN compared to that of SBA-15-APTES can be ascribed to a higher hydrophobicity of the material due to the more hydrophobic functional group, which is usually desired in case of organic molecules adsorption from water solution. Moreover, the electron-withdrawing effect of the aromatic ring enhances the positive charge of the ammonium group, increasing the strength of $\mathrm{H}$-bonding with sugar molecules.

Experimental tests indicated that $\mathrm{pH} 2.1$ is the optimal $\mathrm{pH}$ value to achieve the highest sugars adsorption in a QuEChERS application.

\subsection{Organically modified mesoporous silicas in the d-SPE cleanup of QuEChERS}

In a QuEChERS procedure, after extraction with acetonitrile, a clean-up step performed in $d$-SPE is necessary to remove coextracted interferents without losing analytes of interest. 
a

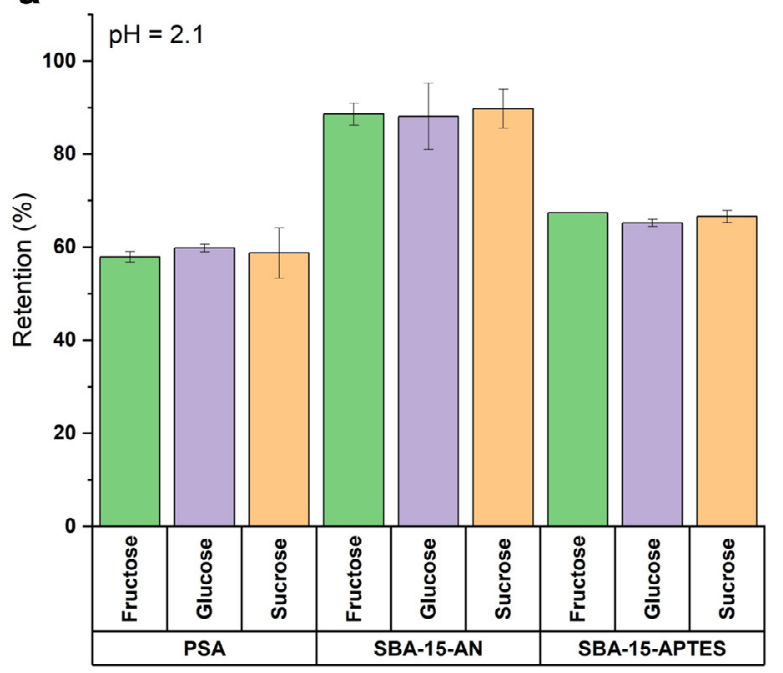

b

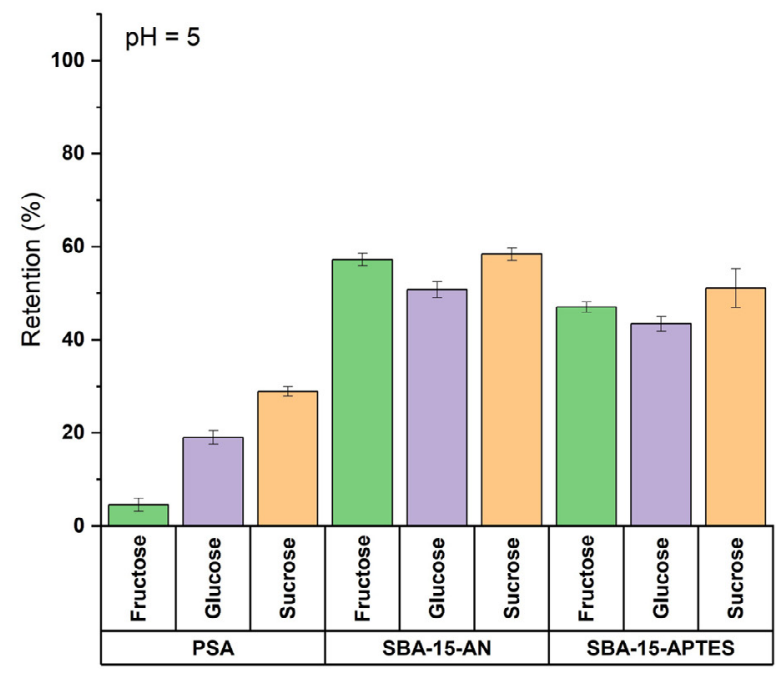

C

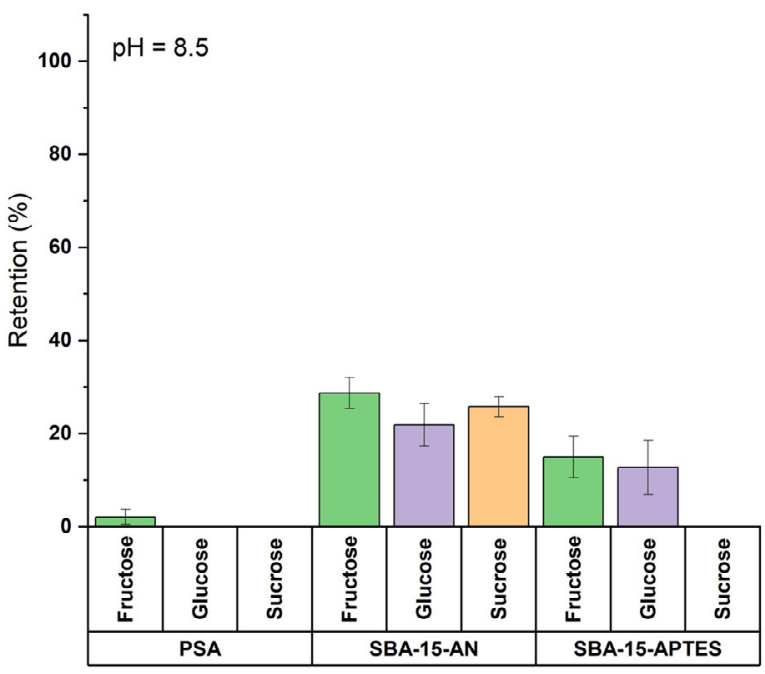

Fig. 3. Adsorption of glucose, fructose, sucrose on PSA, SBA-15-APTES and SBA-15-AN at pH 2.1 (a), 5.0 (b) and 8.5 (c). For experimental details, see text.

To this purpose, the capabilities of SBA-15-APTES and SBA-15AN as $d$-SPE sorbents were investigated in the determination of persistent pollutants (PAHs and PCBs, included dioxine-like congeners) in strawberries, using the QuEChERS extraction approach.

Performances of the modified silicas were studied and compared with those of PSA, measuring both signal-to-noise ratio $(\mathrm{S} / \mathrm{N})$ and extraction recovery (ER) of PAHs and PCBs after the application of the QuEChERS protocol.

$\mathrm{S} / \mathrm{N}$ is a parameter characteristic of the chromatogram and indicates the quantification accuracy of the components during the analytical separation. The higher the $\mathrm{S} / \mathrm{N}$, the better recognized the analyte and the lower the detection limits obtainable. Due to this intrinsic property, $\mathrm{S} / \mathrm{N}$ is considered a primary standard for comparison of chromatographic performances [32] and it is therefore frequently used as response in analytical design optimizations [3234]. In a complex matrix, such as strawberry rich in sugars, anthocyanins and polyphenols [35], S/N is indicative of the efficiency of matrix removal.

Additionally, ER was also measured, since it is indicative of the efficiency of the whole method (extraction and cleanup) to extract analytes of interest, without losing them by adsorption during the clean-up step.

Results obtained by our study for $\mathrm{S} / \mathrm{N}$ are reported in Fig. 4 (PAHs) and 5 (PCBs) while ER values are shown in Fig. 6 (PAHs) and 7 (PCBs). Data obtained in the absence of the d-SPE treatment were also considered.

For an easier interpretation, for each sorbent, experimental results for PAHs are shown, in Fig. 4 and 6, as single value or as average for compounds: up to four benzene rings (acenaphtylene, the isomers: phenathrene/anthracene; fluorene/pyrene; benzo[a]anthracene/chrysene); four benzene rings around a 5-membered ring and five benzene rings (the isomers: benzo[b]fluoranthene/benzo[k]fluoranthene/benzo[a]pyrene);

five benzene rings or five benzene rings around a 5-membered ring (dibenzo[a,h]anthracene and the isomers indeno[1,2,3cd]pyrene/benzo[ghi]perylene). For PCBs, for each sorbent, experimental results were shown, in Fig. 5 and 7, as single value or as average for the 14 congeners tested.

As regards $\mathrm{S} / \mathrm{N}$ values, for all the analytes (Fig. 4 and 5), the values obtained without a $d$-SPE treatment are the lowest, indicating a poor quality of the analytical method. The increase of $\mathrm{S} / \mathrm{N}$ values in the presence of the $d$-SPE treatment indicates a better detectability, essentially due to the removal of coextracted matrix compounds, and the consequent improvement of the baseline. In this regard, both the organically modified mesoporous silica are effective in improving the analytical method.

For PAHs (Fig. 4), S/N obtained with PSA are higher than those obtained with SBA-15-APTES and SBA-15-AN for all the 


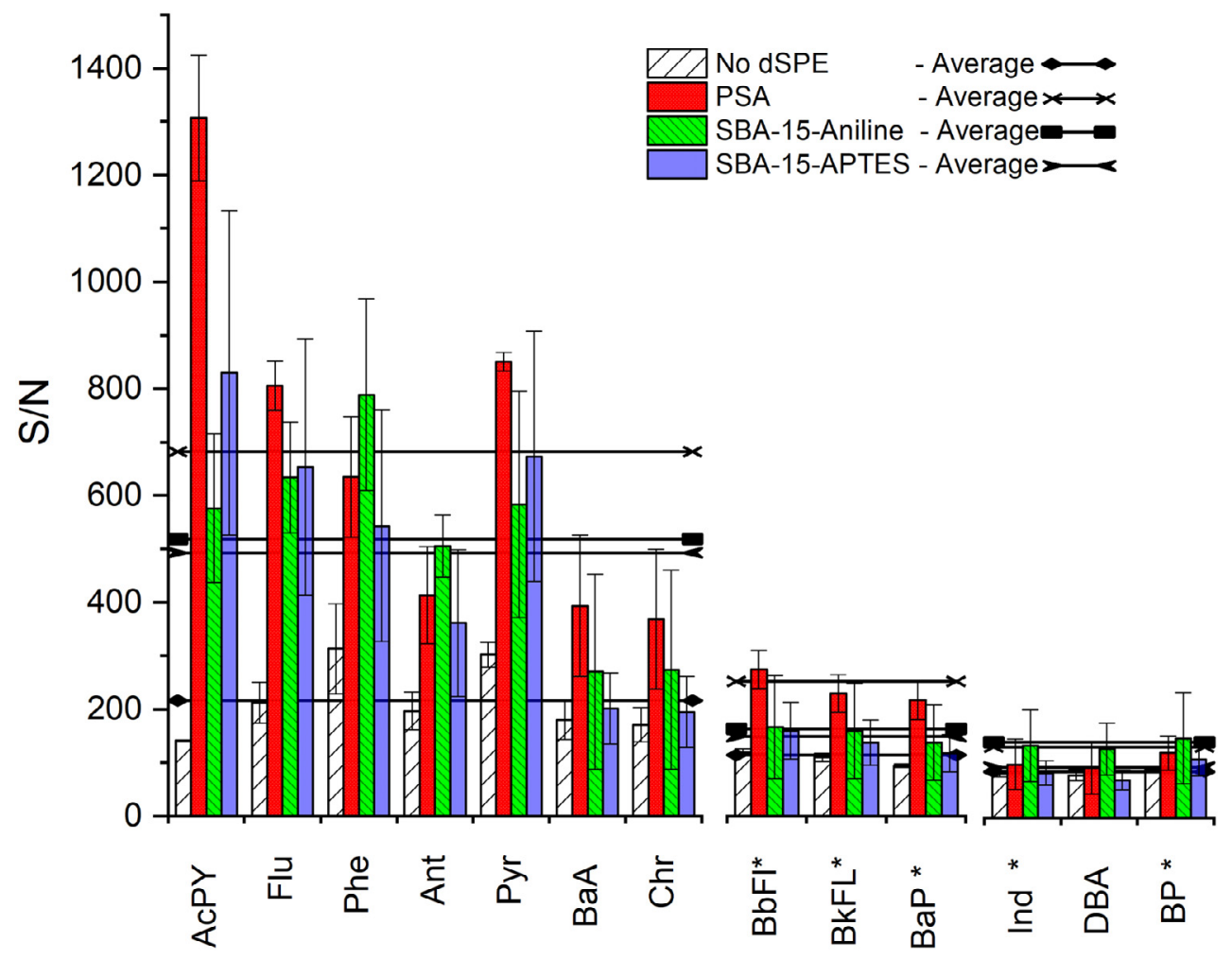

Fig. 4. Signal-to-noise ratio $(\mathrm{S} / \mathrm{N})$ obtained after the QuEChERS extraction of PAHs from strawberry, without and with the d-SPE clean-up by PSA, SBA-15-APTES and SBA-15AN. For QuEChERS extraction conditions, see text.

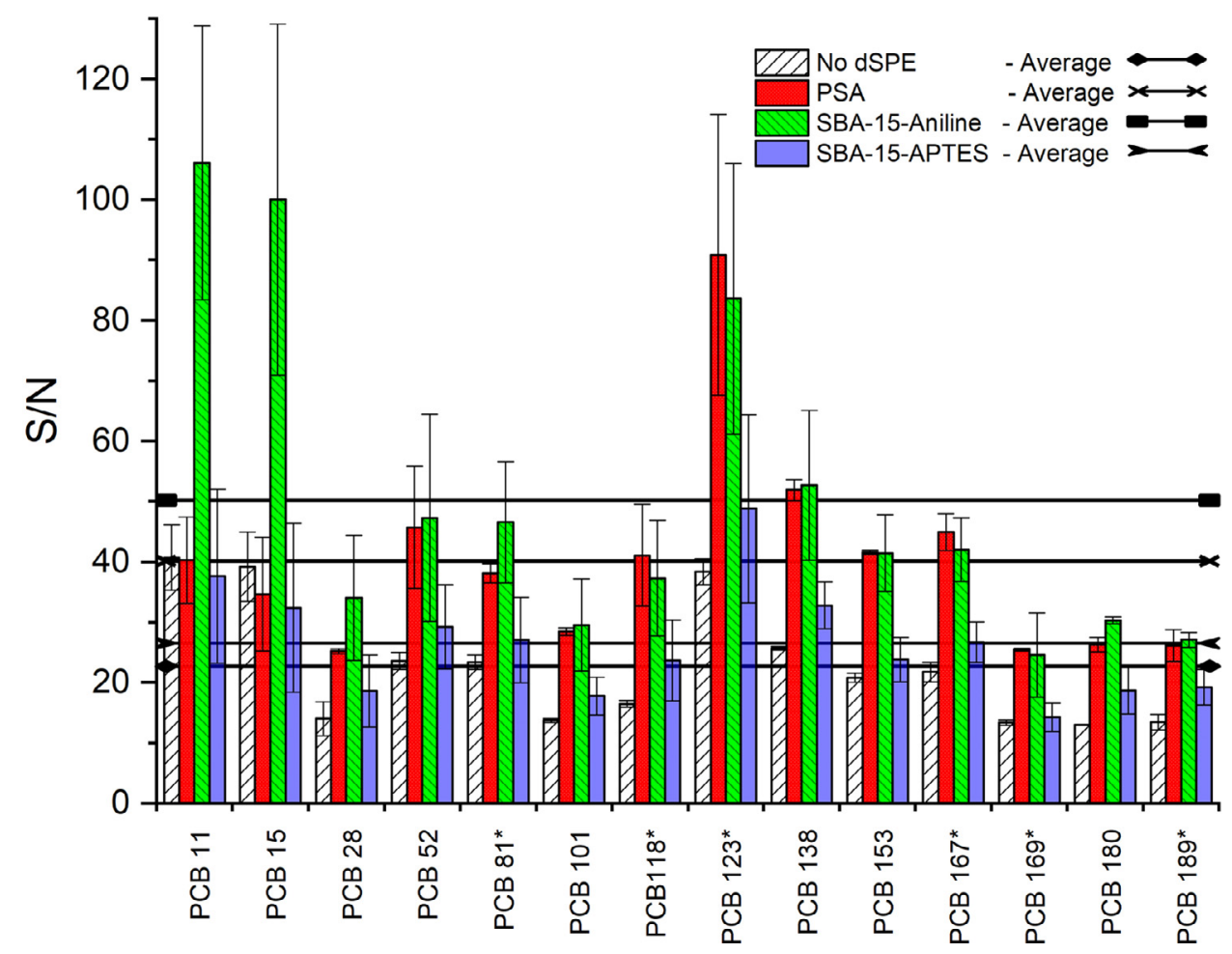

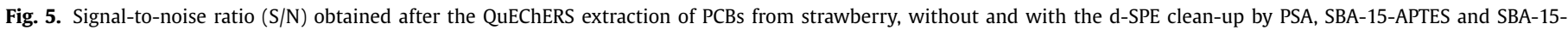
AN. For QuEChERS extraction conditions, see text. 


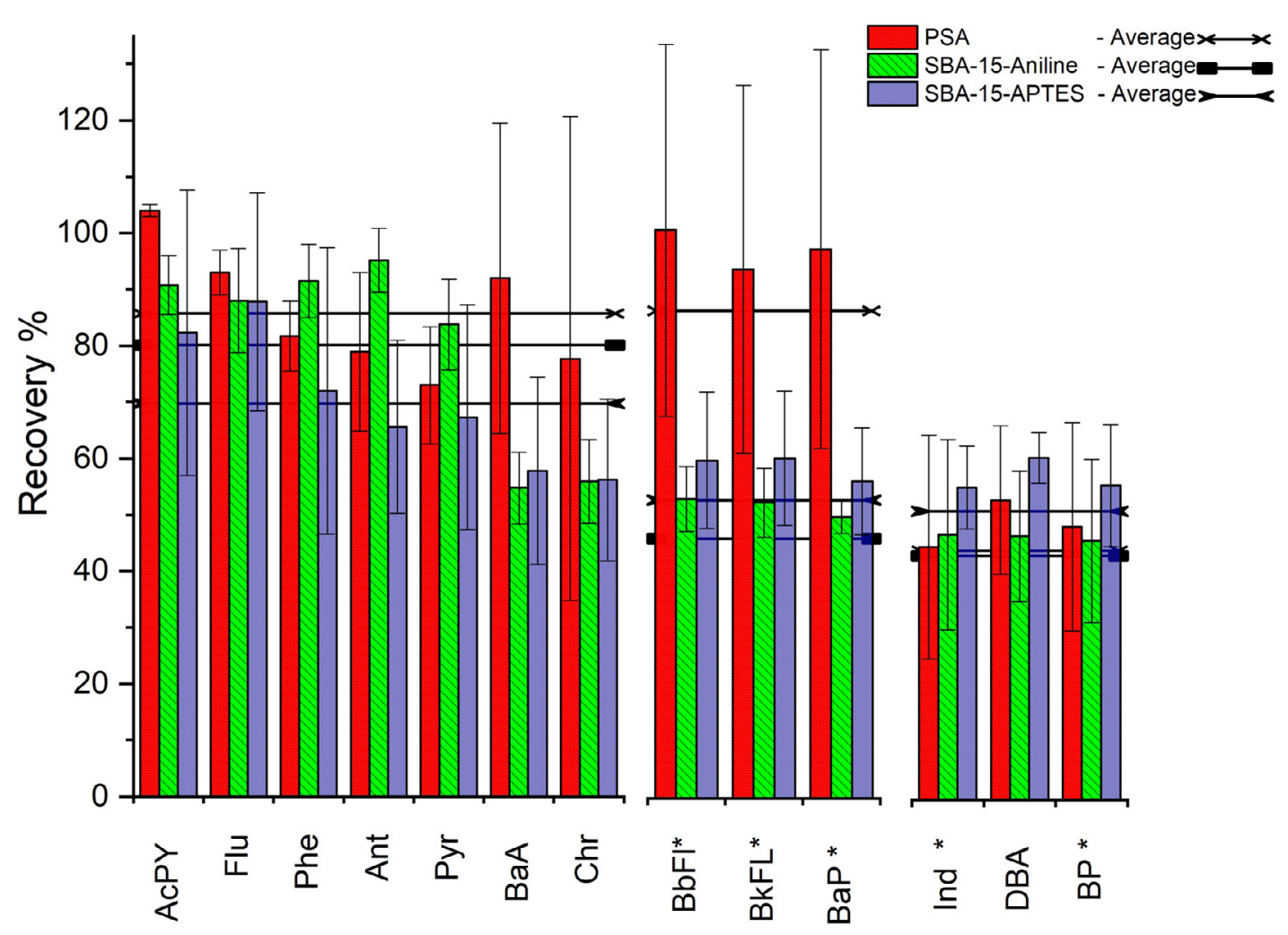

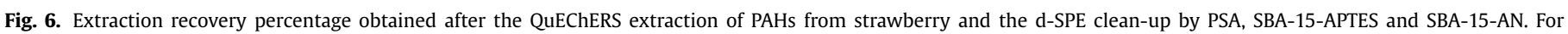
QuEChERS extraction conditions, see text.

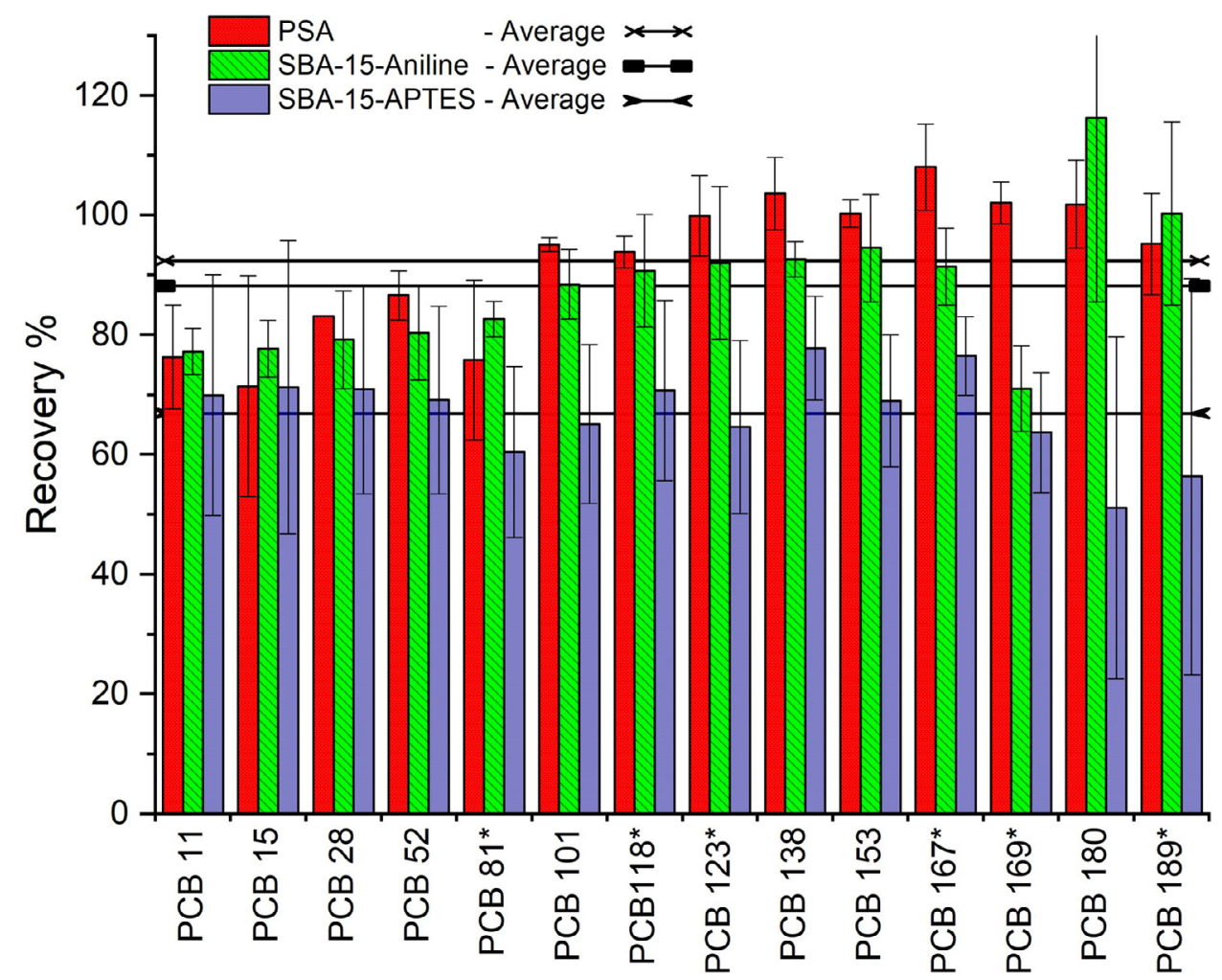

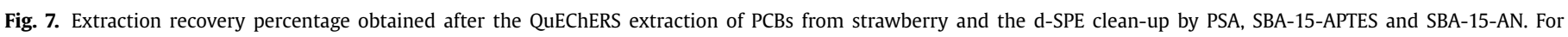
QuEChERS extraction conditions, see text. 

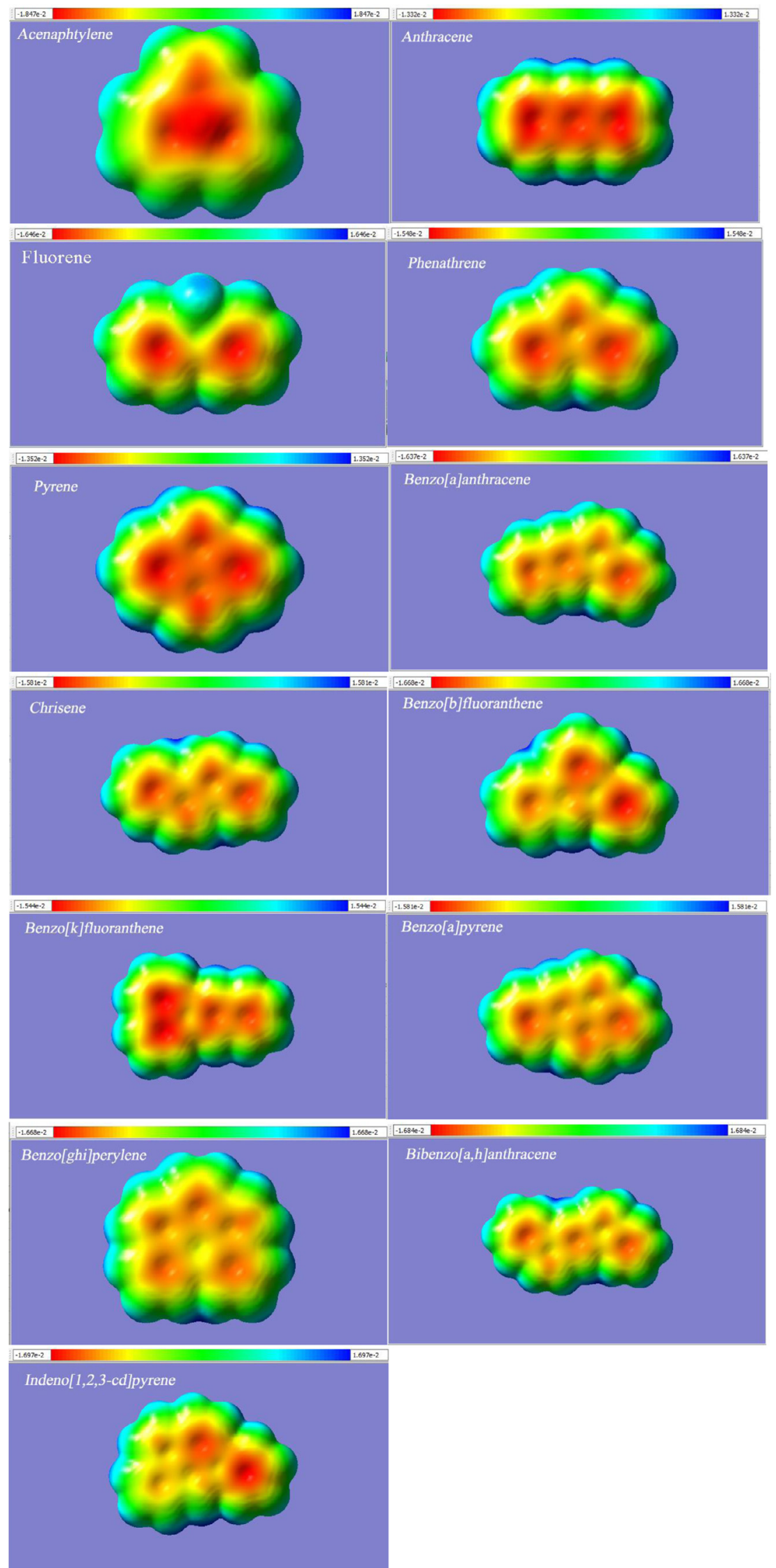

Fig. 8. Electrostatic potential and charge density distribution results of target PAHs, listed following the classes described in paragraph 3.3 (ring classification).

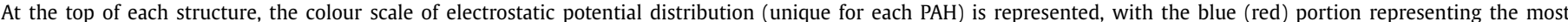
positive (negative) potential. 
hydrocarbons, except for the last three heaviest compounds for which SBA-15-AN is the best performing d-SPE sorbent. The improvement of $\mathrm{S} / \mathrm{N}$ for these last compounds (about 30\%) turns to be important in the enhancement of the detection limits in real matrices, since the heaviest compounds are affected by poor detectability.

For PCBs (Fig. 5), the highest S/N is observed for SBA-15-AN, for which, on average, an improvement of $S / N$ values of about $20 \%$ in respect to PSA is observed.

Extraction recovery ER\% as a function of the d-SPE sorbent used is shown in Fig. 6 for PAHs and in Fig. 7 for PCBs.

For the 13 PAHs, average ER\% follows the order PSA (76 $\pm 19 \%)$ $>$ SBA-15-AN $(63 \pm 7 \%)>$ SBA-15-APTES (61 $\pm 14 \%)$, even though comparable performances are observed for PSA and SBA-15-AN for lower molecular weight PAHs. Differently, ER\% obtained without any d-SPE sorbent are significantly lower, ranging from $14 \pm 3$ to $25 \pm 2 \%$ (data not shown).

According to the Anova test ( $\mathrm{p}=0.0001,95 \%$ confidence interval), the average ER for PSA is statistically different from SBA-15AN and SBA-15-APTES, whereas average ER for the two organically modified mesoporous silica do not show statistical difference. Despite the higher ER obtained for PSA, the lower standard deviation observed for SBA-15-AN indicates that the d-SPE cleanup with SBA-15-AN is more reliable than the one performed with PSA. Statistic variability of ER\% values for each sorbent was also evaluated through Horwtiz equation (Thompson modification [36]), calculating at first the concentration of each $\mathrm{PAH}$, according to its extraction yields, and finally the maximum acceptable RSD\%. Since the maximum acceptable RSD\% value calculated was $22 \%$, the average RSD\% values observed for PAHs with each sorbent $26.2 \%$ for PSA and $14.2 \%$ for SBA-15-AN) indicate that the use of PSA provided recoveries falling outside the range of acceptability. Differently, the reliability of SBA-15-AN is confirmed since average RSD\% is far below the Horwitz value.

Data shows that for all the $d$-SPE sorbents, a decrease of ER\% is observed with the increase of molecular weight introduced by the aromatic rings. This behaviour was verified for PSA also by Sadowska-Rociek et al [37] in the analysis of PAHs in tea.

Even if, overall, PSA is the best performing sorbent for all PAHs, SBA-15-APTES provides higher ER\% for higher molecular weight PAHs.

For PCBs, average ER\% follows the order PSA (92 $\pm 6 \%)>$ SBA15 -AN $(88 \pm 9 \%)>$ SBA-15-APTES $(67 \pm 17 \%)$. Again, poor recovery is observed without any d-SPE step (ER\% ranging from $36 \pm 6$ to
$44 \pm 7 \%$, data not shown). The average ER values obtained for PSA and SBA-15-AN are not statistically different (Anova test, $\mathrm{p}=0.0001$, 95\% confidence interval), whereas average ER values obtained with SBA-15-APTES statistically differ from those obtained by PSA and SBA-15-AN. Again, the application of the Horwitz equation (calculated $\mathrm{RSD} \%=22 \%$ ), confirmed the statistical acceptability for recoveries obtained with PSA and SBA-15-AN (RSD\%=7.2\% and $10.2 \%$, respectively), but not with SBA-15-APTES (RSD\%=25.8\%).

Presuming that the extraction recovery calculation used compensates for any matrix effect, it is reasonable to assume that differences in extraction recovery values observed among the three $\mathrm{d}$-SPE materials is due to the different extent of adsorption of PAHs and PCBs by each sorbent, being the extraction procedure the same for the three tests.

For each sorbent, PCBs exhibit higher ER\% values than PAHs. For each d-SPE sorbent, for PAHs an increase of ER\% is observed upon decreasing molecular weight (MW), showing a partial anticorrelation (the higher the MW, the lower the ER\%) for SBA-15-AN $\left(\mathrm{ER} \%=-1.9 \mathrm{MW}+346, \mathrm{r}^{2}=0.919\right)$ and for SBA-15-APTES (ER\% = $\left.3.3 \mathrm{MW}+424, \mathrm{r}^{2}=0.840\right)$, while for PSA this behaviour is not well evidenced (ER\% $=-1.5 \mathrm{MW}+340, \mathrm{r}^{2}=0.429$ ). This suggests that at variance with what hypothesized by Dachs and Bayona [38] for silica based octadecyl substrates, in this case, steric hindrance and shape selectivity do not play a crucial role in the extraction. To explain the higher extent of adsorption observed for higher molecular weight PAHs, density functional theory (DFT) simulations were calculated by the software Gaussview 6.0 in order to estimate the potential and charge density distribution for each PAH tested [39].

Results obtained (Fig. 8) show that the centre of each ring on both sides of the PAH molecular plane is negatively charged and corresponds with delocalized $\pi$ electrons. Indeed, the area of the $\pi$-electron plane spatially increases when the number of rings increased, as demonstrated also by Yang and co-workers [40]. Hence, the heavier molecular weight of the PAHs offers higher surface availability for interaction with positively charged amino groups. Moreover, such behaviour is also in agreement with stronger Van der Waals interactions. Conversely, for PCBs, the addition of chlorine atoms on the biphenyl structure enhances steric hindrance, in agreement with observations of Dachs and Bayona [38]. In Fig. 9, as an example, the different behaviour of PAHs (Fig. 9a) and PCBs (Fig. 9b) is compared for SBA-15-AN.

Eventually the polarity of micropollutants was also considered. Variations in $\log \mathrm{P}$ does not significantly influence the extraction performance of the QuEChERS method when SBA-15-APTES or

\section{a}

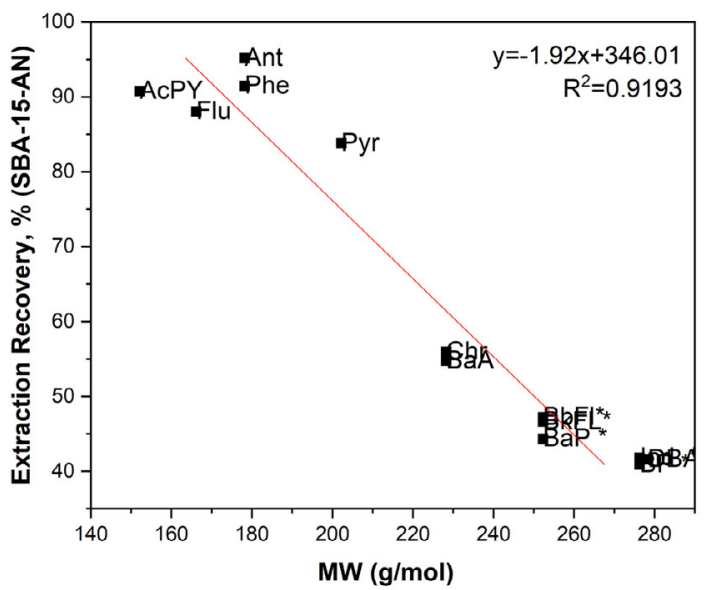

b

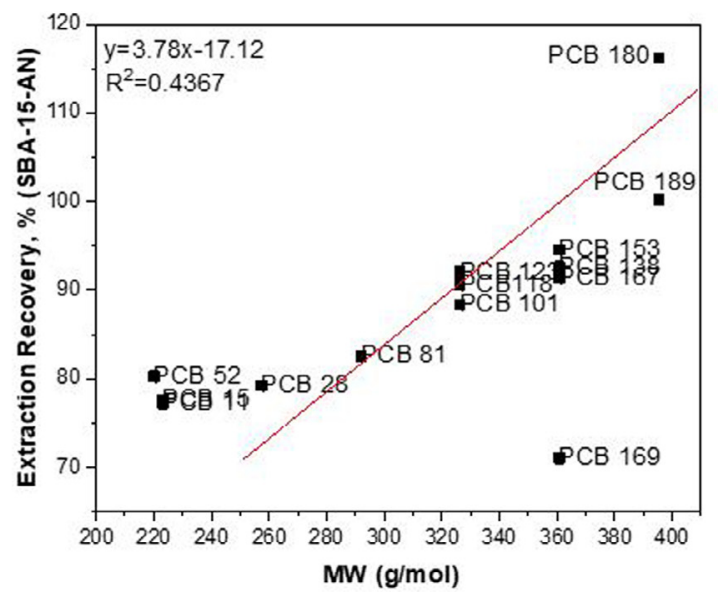

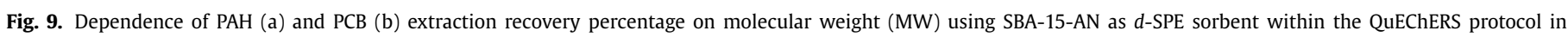
strawberry. 
SBA-15-AN are used as d-SPE sorbents. Instead, an apparent correlation of ER\% with log P for both PAHs and PCBs classes is observed only for PSA $\left(E R \%=10.36 \log \mathrm{P}+0.49, \mathrm{r}^{2}=0.653\right)$, highlighting possible limitations in its use of this d-SPE sorbent for more polar analytes. This observation agrees with what found by Scordo et al. [1] in the extraction of perfluoroalkyl acids from strawberry.

\section{Conclusions}

SBA-15 mesoporous silica were functionalized with (3aminopropyl)-triethoxysilane (SBA-15-APTES) and $\mathrm{N}$-[3(Trimethoxysilyl)propyllaniline (SBA-15-AN) and used for the first time as d-SPE sorbents in the removal of coextracted compounds (e.g. sugars) in the QuEChERS protocol applied for the determination of micropollutants (PAHs and PCBs) in strawberry. SBA-15-APTES and SBA-15-AN were physico-chemically characterized and compared with PSA, the d-SPE sorbent usually employed in QuEChERS application. The removal capabilities of SBA-15-APTES and SBA-15-AN towards glucose, sucrose and fructose, chosen as model sugars present in strawberry was observed to be higher than that of PSA, due to the higher amount of adsorption active sites. A thorough study of the effect of $\mathrm{pH}$ on removal of sugars allowed to propose an interaction mechanism between amines and sugar molecules mainly based on H-bonding.

The suitability of SBA-15-APTES and SBA-15-AN as d-SPE sorbents were confirmed including these sorbents in the clean-up step of a QuEChERS protocol for the determination of PAHs and PCBs in intentionally contaminated strawberries. Signal-to-noise ratio for PCBs can be significantly reduced by SBA-15-AN, indicating even a more efficient cleanup if compared to PSA. It is worth remembering that the reduction of signal-to-noise ratio is important to determine lower concentrations of micropollutants in food. Overall, QuEChERS protocol performed by SBA-15-AN provides slightly lower or comparable extraction recoveries than PSA ( $63 \pm 7 \%$ vs $76 \pm 19 \%$ for PAHs, respectively and $92 \pm 6 \%$ vs $88 \pm 9$ $\%$ for PCBs) and better reproducibility of the method. Molecular weight of the target micropollutants, but not $\log \mathrm{P}$, seems to influence the overall QuEChERS extraction recovery in the organically modified SBA-15.

\section{Author statement}

M. Castiglioni: investigation, writing original draft, manuscript revision. B. Onida: data interpretation, review and manuscript editing. L. Rivoira: supervision, data validation and curation, manuscript revision. M. Del Bubba: data interpretation, manuscript editing. S. Ronchetti: data interpretation, review. M.C. Bruzzoniti: conceptualization, data interpretation, review and manuscript editing, funding acquisition, manuscript revision.

\section{Declaration of Competing Interest}

The authors declare that they have no known competing financial interests or personal relationships that could have appeared to influence the work reported in this paper.

\section{Acknowledgements}

Financial supports from Ministero della Ricerca e dell'Università (MUR, Italy), PRIN 2017 (017PMR932) and Ex-60\% are gratefully acknowledged.

\section{Supplementary materials}

Supplementary material associated with this article can be found, in the online version, at doi:10.1016/j.chroma.2021.462107.

\section{References}

[1] C.V.A. Scordo, L. Checchini, L. Renai, S. Orlandini, M.C. Bruzzoniti, D. Fibbi, L. Mandi, N. Ouazzani, M. Del Bubba, Optimization and validation of a method based on QuEChERS extraction and liquid chromatographic-tandem mass spectrometric analysis for the determination of perfluoroalkyl acids in strawberry and olive fruits, as model crops with different matrix characteristics, J. Chromatogr. A 1621 (2020) 461038, doi:10.1016/j.chroma.2020. 461038.

[2] L. Rivoira, M. Castiglioni, A. Kettab, N. Ouazzani, E. Al-Karablieh, N. Boujelben, D. Fibbi, E. Coppini, E. Giordani, M.D. Bubba, M.C. Bruzzoniti, Impact of effluents from wastewater treatments reused for irrigation: Strawberry as case study, Environmental Engineering and Management Journal 18 (10) (2019) $2133-2143$.

[3] K. Grob, M. Biedermann, E. Scherbaum, M. Roth, K. Rieger, Food contamination with organic materials in perspective: packaging materials as the largest and least controlled source? A view focusing on the European situation, Critical reviews in food science and nutrition 46 (7) (2006) 529-535, doi:10.1080/ 10408390500295490.

[4] L. Sung-Jin, O. Young-Tak, J. You-Sung, R. Jin-Ho, C. Geun-Hyoung, Y. Ji-Yeon P. Byung-Jun, Persistent organic pollutants (POPs) residues in greenhouse soil and strawberry organochlorine pesticides, Korean Journal of Environmental Agriculture 35 (1) (2016) 6-14 doi:10.5338/KJEA.2016.35.1.05.

[5] European Commission, 2014/663/EU: Commission Recommendation of 11 September 2014 amending the Annex to Recommendation 2013/711/EU on the reduction of the presence of dioxins, furans and PCBs in feed and food Text with EEA relevance, (2014)

[6] M. Anastassiades, S.J. Lehotay, D. Stajnbaher, F.J. Schenck, Fast and easy multiresidue method employing acetonitrile extraction/partitioning and "dispersive solid-phase extraction" for the determination of pesticide residues in produce, J. AOAC Int. 86 (2) (2003) 412-431, doi:10.1093/jaoac/86.2.412.

[7] D. Rossini, L. Ciofi, C. Ancillotti, L. Checchini, M.C. Bruzzoniti, L. Rivoira, D. Fibbi, S. Orlandini, M. Del Bubba, Innovative combination of QuEChERS extraction with on-line solid-phase extract purification and pre-concentration, followed by liquid chromatography-tandem mass spectrometry for the determination of non-steroidal anti-inflammatory drugs and their metabolites in sewage sludge, Anal. Chim. Acta 935 (2016) 269-281, doi:10.1016/j.aca.2016. 06.023.

[8] M.C. Bruzzoniti, L. Checchini, R.M. De Carlo, S. Orlandini, L. Rivoira, M. Del Bubba, QuEChERS sample preparation for the determination of pesticides and other organic residues in environmental matrices: a critical review, Anal Bioanal Chem 406 (17) (2014) 4089-4116, doi:10.1007/s00216-0147798-4.

[9] D. Oshita, I.C.S.F. Jardim, Comparison of Different Sorbents in the QuEChERS Method for the Determination of Pesticide Residues in Strawberries by LC-MS/MS, Chromatographia 77 (19) (2014) 1291-1298, doi:10.1007/ s10337-014-2726-5.

[10] Y. He, Y.-H. Liu, Assessment of primary and secondary amine adsorbents and elution solvents with or without graphitized carbon for the SPE clean-up of food extracts in pesticide residue analysis, Chromatographia 65 (9-10) (2007) 581-590, doi:10.1365/s10337-007-0198-6.

[11] Z. Wu, D. Zhao, Ordered mesoporous materials as adsorbents, Chem. Commun. 47 (12) (2011) 3332-3338, doi:10.1039/C0CC04909C

[12] C.M. Li, X.P. Wang, Z.H. Jiao, Y.S. Zhang, X.B. Yin, X.M. Cui, Y.Z. Wei, Functionalized Porous Silica-Based Nano/Micro Particles for Environmental Remediation of Hazard Ions, Nanomaterials 9 (2) (2019) 247, doi:10.3390/nano9020247.

[13] P. Kumar, V.V. Guliants, Periodic mesoporous organic-inorganic hybrid materials: applications in membrane separations and adsorption, Microporous Mesoporous Mater 132 (1-2) (2010) 1-14, doi:10.1016/j.micromeso.2010. 02.007.

[14] M.C. Bruzzoniti, E. Mentasti, C. Sarzanini, B. Onida, B. Bonelli, E. Garrone, Retention properties of mesoporous silica-based materials, Anal. Chim. Acta 422 (2) (2000) 231-238, doi:10.1016/S0003-2670(00)01070-9.

[15] N. Casado, D. Pérez-Quintanilla, S. Morante-Zarcero, I. Sierra, Current development and applications of ordered mesoporous silicas and other sol-gel silicabased materials in food sample preparation for xenobiotics analysis, TrAC, Trends Anal. Chem. 88 (2017) 167-184, doi:10.1016/j.trac.2017.01.001.

[16] S. Fiorilli, L. Rivoira, G. Calì, M. Appendini, M.C. Bruzzoniti, M. Coïsson, B. Onida, Iron oxide inside SBA-15 modified with amino groups as reusable adsorbent for highly efficient removal of glyphosate from water, Appl. Surf. Sci. 411 (2017) 457-465, doi:10.1016/j.apsusc.2017.03.206.

[17] L. Rivoira, M. Appendini, S. Fiorilli, B. Onida, M. Del Bubba, M.C. Bruzzoniti, Functionalized iron oxide/SBA-15 sorbent: investigation of adsorption performance towards glyphosate herbicide, Environmental Science and Pollution Research 23 (21) (2016) 21682-21691, doi:10.1007/s11356-016-7384-8.

[18] A. Paris, J. Ledauphin, P. Poinot, J.-L. Gaillard, Polycyclic aromatic hydrocarbons in fruits and vegetables: Origin, analysis, and occurrence, Environ. Pollut. 234 (2018) 96-106, doi:10.1016/j.envpol.2017.11.028.

[19] A.A. Lovett, C.D. Foxall, C.S. Creaser, D. Chewe, PCB and PCDD/DF congeners in locally grown fruit and vegetable samples in Wales and England, Chemosphere 34 (5) (1997) 1421-1436, doi:10.1016/S0045-6535(97)00439-6.

[20] M.C. Bruzzoniti, L. Rivoira, M. Castiglioni, A. El Ghadraoui, A. Ahmali, T. El Hakim El Mansour, L. Mandi, N. Ouazzani, M. Del Bubba, Extraction of polycyclic aromatic hydrocarbons and polychlorinated biphenyls from urban and olive mill wastewaters intended for reuse in agricultural irrigation, J. AOAC Int. 103 (2) (2020) 382-391, doi:10.5740/jaoacint.19-0257. 
[21] M.J. Kasperbauer, J.H. Loughrin, S.Y. Wang, Light Reflected from Red Mulch to Ripening Strawberries Affects Aroma, Sugar and Organic Acid Concentrations, Photochem. Photobiol. 74 (1) (2001) 103-107, doi:10.1562/0031-8655(2001) 0740103LRFRMT2.0.CO2.

[22] C.H. Vane, A.W. Kim, D.J. Beriro, M.R. Cave, K. Knights, V. Moss-Hayes, P.C. Nathanail, Polycyclic aromatic hydrocarbons (PAH) and polychlorinated biphenyls (PCB) in urban soils of Greater London, UK, Appl. Geochem. 51 (2014) 303-314, doi:10.1016/j.apgeochem.2014.09.013.

[23] R.M. De Carlo, L. Rivoira, L. Ciofi, C. Ancillotti, L. Checchini, M. Del Bubba, M.C. Bruzzoniti, Evaluation of different QuEChERS procedures for the recovery of selected drugs and herbicides from soil using LC coupled with UV and pulsed amperometry for their detection, Analytical and Bioanalytical Chemistry 407 (4) (2015) 1217-1229, doi:10.1007/s00216-014-8339-x.

[24] United States of Pharmacopoeia 38 NF 33, in: United States Pharmacopeial Convention,General chapter $<621>$, 2015, pp. 424-434.

[25] D. Brunel, A.C. Blanc, E. Garrone, B. Onida, M. Rocchia, J.B. Nagy, D.J. Macquarrie, Spectroscopic studies on aminopropyl-containing micelle templated silicas. Comparison of grafted and co-condensation routes, in: R. Aiello, G. Giordano, F. Testa (Eds.), Stud. Surf. Sci. Catal., Elsevier, 2002, pp. 1395-1402, doi:10.1016/S0167-2991(02)80305-6.

[26] B. Onida, L. Borello, C. Busco, P. Ugliengo, Y. Goto, S. Inagaki, E. Garrone, The surface of ordered mesoporous benzene - Silica hybrid material: An infrared and ab initio molecular modeling study, J. Phys. Chem. B 109 (24) (2005) 11961-11966, doi:10.1021/jp050686n.

[27] Y.Y. M. Ye, A. Trinh, Analysis of Multi-Pesticide Residues in Vegetables Food, and Fruits by SPE/GC-MS, 2020. available at https://www.sigmaaldrich.com/ content/dam/sigma-aldrich/docs/Supelco/Posters/t405020h.pdf last accessed September.

[28] O. Shimelis, Y. Yang, K. Stenerson, T. Kaneko, M. Ye, Evaluation of a solid-phase extraction dual-layer carbon/primary secondary amine for clean-up of fatty acid matrix components from food extracts in multiresidue pesticide analysis, J. Chromatogr. A 1165 (1) (2007) 18-25, doi:10.1016/j.chroma.2007.07.037.

[29] F. Plössl, M. Giera, F. Bracher, Multiresidue analytical method using dispersive solid-phase extraction and gas chromatography/ion trap mass spectrometry to determine pharmaceuticals in whole blood, J. Chromatogr. A 1135 (1) (2006) 19-26, doi:10.1016/j.chroma.2006.09.033.

[30] D. Orso, M. Martins, F. Donato, T. Rizzetti, M. Kemmerich, M. Adaime, R. Zanella, Multiresidue Determination of Pesticide Residues in Honey by Modified QuEChERS Method and Gas Chromatography with Electron Capture Detection, Journal of the Brazilian Chemical Society 25 (2014), doi:10.5935/ 0103-5053.20140117.
[31] C. Ling, X. Li, Z. Zhang, F. Liu, Y. Deng, X. Zhang, A. Li, L. He, B. Xing, High adsorption of sulfamethoxazole by an amine-modified polystyrenedivinylbenzene resin and its mechanistic insight, Environmental science \& technology 50 (18) (2016) 10015-10023, doi:10.1021/acs.est.6b02846.

[32] G. Wells, H. Prest, C.W. Russ IV, Why use signal-to-noise as a measure of MS performance when it is often meaningless?, Technical Overview, Agilent Technologies, 2011 available at https://www.google.com/url?sa =t\&rct=j\&q=\&esrc= $\mathrm{s} \&$ source $=$ web $\& \mathrm{~cd}=\& \mathrm{cad}=\mathrm{rja} \& u a c t=8 \&$ ved $=2$ ahUKEwiN_76uuJPuAhVPCuwKHY _cDTUQFjABegQIBxAC\&url=https\%3A\%2F\%2Fwww.agilent.com\%2Fcs\%2Flibrary\% 2Ftechnicaloverviews\%2Fpublic\%2F5990-8341EN.pdf\&usg=AOvVaw35pJ2yCw Yqtgeugpe9yszj.

[33] J. Bérubé, C. Wu, Signal-to-noise ratio and related measures in parameter design optimization: an overview, Sankhyā: The Indian Journal of Statistics, Series B (2000) 417-432.

[34] L. Rivoira, R.M. De Carlo, S. Cavalli, M.C. Bruzzoniti, Simple SPE-HPLC determination of some common drugs and herbicides of environmental concern by pulsed amperometry, Talanta 131 (2015) 205-212, doi:10.1016/j.talanta.2014.07. 070.

[35] L. Renai, F. Tozzi, C.V. Scordo, E. Giordani, M.C. Bruzzoniti, D. Fibbi, L. Mandi, N. Ouazzani, M. Del Bubba, Productivity and nutritional and nutraceutical value of strawberry fruits (Fragaria $\mathrm{x}$ ananassa Duch.) cultivated under irrigation with treated wastewaters, J. Sci. Food Agric. (2020) in press, doi:10.1002/ jsfa.10737.

[36] C. Rivera, R. Rodríguez, Horwitz equation as quality benchmark in ISO/IEC 17025 testing laboratory, Private communication (2014).

[37] A. Sadowska-Rociek, M. Surma, E. Cieślik, Comparison of different modifications on QuEChERS sample preparation method for PAHs determination in black, green, red and white tea, Environmental Science and Pollution Research 21 (2) (2014) 1326-1338, doi:10.1007/s11356-013-2022-1.

[38] J. Dachs, J.M. Bayona, Large volume preconcentration of dissolved hydrocarbons and polychlorinated biphenyls from seawater. Intercomparison between C18 disks and XAD-2 column, Chemosphere 35 (8) (1997) 1669-1679, doi:10. 1016/S0045-6535(97)00248-8.

[39] T. Sato, T. Tsuneda, K. Hirao, A density-functional study on $\pi$-aromatic interaction: Benzene dimer and naphthalene dimer, The Journal of chemical physics 123 (10) (2005) 104307, doi:10.1063/1.2011396.

[40] X. Yang, C. Zhang, L. Jiang, Z. Li, Y. Liu, H. Wang, Y. Xing, R.T. Yang, Molecular simulation of naphthalene, phenanthrene, and pyrene adsorption on MCM41, International journal of molecular sciences 20 (3) (2019) 665, doi:10.3390/ ijms20030665. 